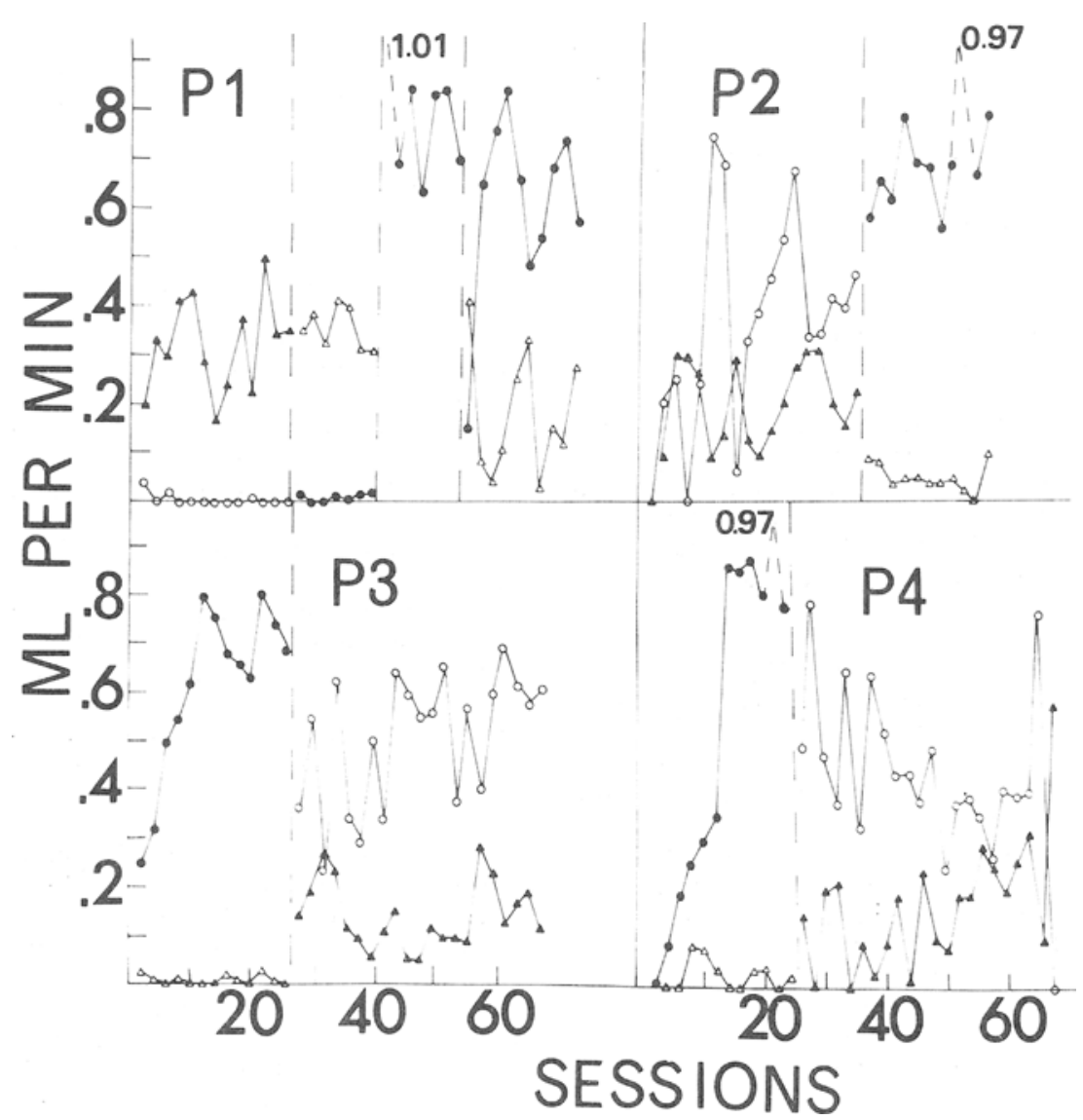

Fig. 1. Rates of liquid consumption for each $\mathrm{S}$ a veraged over two sessions. The triangles and circles represent alcohol and water, respectively. Filled symbols are for the reinforced liquid, and open symbols are for the nonreinforced liquid.

\section{DISCUSSION}

The main point of the present experiment is that alcohol consumption was increased by reinforcing it with an increase in the frequency of pellet delivery under the dpr contingency, even when a pellet could not follow alcohol drinking by less than 3 sec. These results decrease the probability that the increased alcohol consumption observed by Keehn \& Coulson (1970) was due to adventitious reinforcement.

The observation that three of the four Ss drank more water than alcohol, even when alcohol was the reinforced liquid, suggests a preference for water; a similar preference for water was noted by Keehn and Coulson. In spite of this preference, the drinking pattern of the aberrant $S$, $P-1$, indicates that, in some cases, it might be necessary to take special steps, such as reinforcing water drinking in the absence of alcohol, in order to reverse a preference for alcohol induced by a dpr schedule of reinforcement.
Figure 1 shows the average fluid intake in milliliters per minute, averaged over two sessions for individual $S s$ as a function of session number and contingency.

The development of a strong preference for alcohol under the dpr schedule employed in this experiment is seen most clearly in the drinking pattern for P-1. During Phase 2, when the reinforced liquid was changed from alcohol to water, P-1 continued to drink alcohol almost exclusively. When only water was available as the reinforced liquid for 14 sessions, the amount of water consumed by P-1 increased and was maintained upon the subsequent reintroduction of the alcohol bottle.

The consummatory behavior of P-2 was under control of the dpr schedule; water drinking increased and alcohol drinking decreased when the reinforced liquid was switched from alcohol to water. P-3 and P-4 both exhibited a strong preference for water, even in the absence of reinforcement for water drinking. However, they both increased their alcohol consumption when alcohol drinking was reinforced.

\title{
Schedule control in the White-Necked Raven,
} Corvus cryptoleucus*

\author{
R. R. HANEY, JOHN A. BEDFORD, and ROBERT BERRYMAN \\ The University of Mississippi, University, Miss. 38677
}

Five White-Necked ravens were run to behavioral stability on each of three moderate schedules of positive reinforcement: FR 50, FI $4 \mathrm{~min}$, and RI $2.5 \mathrm{~min}$. The sequence of schedule exposure for any one raven was different from that for any other raven. Temporal variations in rates emitted by the raven under these schedules were characteristic of those emitted by other species more typically used in operant conditioning studies.

Behavior theorists generally assume that the major effects of positive

\footnotetext{
*This research was sponsored by the Air Force Office of Scientific Research (AFSC). United States Air Force, Contract No. F 44620-69-C-0050. Portions of these data were presented at the Southeastern Psychological Association Meeting. Louisville, Ky., April 24-26, 1970. Reprints may be obtained from R. R. Haney, Department of Psychology, The University
} of Mississippi. University, Miss. 38677. reinforcement schedules are species independent. Indeed, a number of studies (e.g., Skinner, 1961) have shown that species membership is perhaps one of the least important determinants of schedule performance. However, it is standard practice to test this assumption when one first applies operant conditioning methods to a new species.

Since ancient times (cf. I Kings 17:6), man has considered members of 


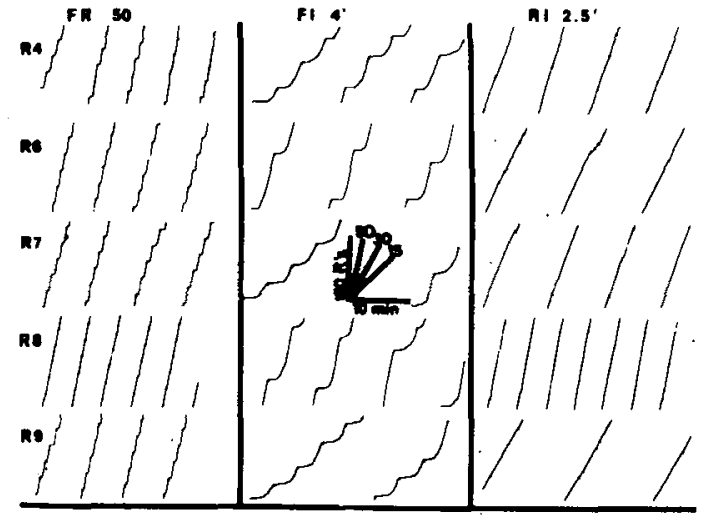

Fig. 1. Representative cumulative records for each schedule and $\mathrm{S}$ after stability criteria had been met.

the crow family to possess "mental qualities of a high order" (Austin, 1961) compared to other bird species. If there are objective grounds for these beliefs, corvines might well be useful in studies of complex operant discriminations. However, there are few relevant investigations using such Ss. Koehler (1950) and Hassman (1952), using matching-to-sample procedures, demonstrated learning in the raven. Koehler's birds, when presented with a "key" card and five comparison boxes, lifted the lid of the box with the same number of dots as appeared on the card. Hassman's "standard" stimulus cards had varying numbers of objects or marks, and the "comparison" boxes had varying numbers of dots. His raven also matched the number of dots on the box lids to the number of objects on the standard card and lifted the lid of the appropriate box.

As a preliminary step in a projected series of experiments on complex operant discriminations, we studied the effects of fixed-ratio, fixed-interval, and random-interval schedules on the behavior of the White-Necked raven, Corvus cryptoleucus.

\section{SUBJECTS}

Six experimentally naive White-Necked ravens, about 1.5 years old, were obtained as wild-bred fledglings and maintained at approximately $80 \%$ of their free-feeding weights throughout the study. Reinforcements and maintenance rations were commercial mynah bird food pellets. 1 Water and grit were available at all times in the individual home cages.

\section{APPARATUS}

The experimental chamber measured $406 \times 406 \times 406 \mathrm{~mm}$ and was enclosed in a sound-attenuating chamber. On the intelligence panel was a single key, $25 \mathrm{~mm}$ in diam, chamber floor, and transilluminated with white light. Reinforcement consisted of 3-sec access to pellets delivered by a pigeon feeder (LVE No. 1347) through a 51-mm-diam aperture centered $89 \mathrm{~mm}$ from the floor. The hopper was modified by increasing the opening by $6 \mathrm{~mm}$ on all sides to accommodate the raven's beak, which is considerably larger than the pigeon's. Remote solid-state programming and recording equipment was used.

\section{PROCEDURE}

Following manual shaping of magazine approach and keypecking on continuous reinforcement, Ss were exposed to one of the following schedules: fixed-ratio 50 (FR 50), fixed-interval $4 \mathrm{~min}$ (FI $4 \mathrm{~min}$ ), and random-interval $2.5 \mathrm{~min}$ (RI $2.5 \mathrm{~min}$ ). Each raven was assigned randomly to one of the six possible permutation orders for sequential presentation of the three schedules. These orders were: R-4-FI, FR, RI; R-6-RI, FI, FR; R-7-FR, RI, FI; R-8-RI, FR, FI; R-9-FR, FI, RI. R-5 had been assigned to the sequence, FI, RI, FR, but died before generating any useful data.

The birds were shifted to a new schedule only after meeting a stability criterion. For the interval schedules, this was the index used by Schoenfeld, Cumming, \& Hearst (1956), while, for the ratio schedule, stability was determined by visual inspection of the cumulative records and the requirement that response rates did not change noticeably over seven consecutive sessions.

For FR, sessions were terminated after 50 reinforcements, for FI, after 20 intervals, and for RI, after $1 \mathrm{~h}$. center-mounted $305 \mathrm{~mm}$ from the
RESULTS AND DISCUSSION

Manual shaping of the keypeck by the method of successive approximation was easily achieved with all Ss.

Final performances, after stability criteria had been met, are shown in Fig. 1 for all $S s$ and all schedule conditions. Schedule control was quite precise, and the observed effects are highly similar to those described by Ferster \& Skinner (1957) for FI and FR schedules, by Millenson (1963) for the RI schedule, and elsewhere in the literature. The FR contingency, as might be expected (cf. Berryman \& Nevin, 1962), produced the least inter-S variability. The FI and RI contingencies, in contrast, produced marked rate differences from bird to bird, although the characteristic major schedule effects scalloping on FI and sustained generally high rates on $\mathrm{RI}-$ were present in all Ss.

Presumably, some of these individual differences are a function of the sequence of schedule presentation. $R-8$, for example, with the sequence RI-FR-FI, had the highest rate of any $S$ on RI, was the only bird not to show any significant amount of postreinforcement pausing on FR, and worked at quite high rates on FI. This makes at least some measure of sense in terms of the initial exposure to RI contingencies carrying over to the FR and FI schedules. However, for other Ss the effects are by no means simple. R-4, whose initial exposure was to FI (FI-FR-RI), had a low overall rate on this schedule; but so did R-7, with the sequence FR-RI-FI.

While the data are insufficient for the analysis of these sequential effects, we can report that schedule control in the raven is highly similar to that observed in a wide variety of human and animal Ss.

\section{REFERENCES}

AUSTIN, O. L. Binds of the world. New Y ork: Golden Press, 1961

BERRYMAN, R., \& NEVIN, J. A. Interlocking schedules of reinforcement Journal of the Experimental Analysis of Behavior, 1962, 5, 213-223.

FERSTER, C. B., \& SKINNER, B, F. Schedules of reinforcement. New York: Appleton-Century-Crofts, 1957.

HASSM AN, M. Vom erlernen unbenannter Anzahlen bei Eichornchen. Z eitschrift für Tierspsy chologie, 1952, 9, 294-321.

KOEHLER, $O$. The ability of birds to count. Bulletin of Animal Behaviour, $1950,9,41-45$.

MILLENSON, J. R. Random interval schedules of reinforcement. Journal of the Experimental Analysis of Behavior. $1963,6,437-443$.

SCHOENFELD, W. N., CUMMING. W. W.. \& HEARST, E. On the classification of reinforcement schedules. National Academy of Sciences, 1956, 42, 563-570. SKINNER, B. F. Cumulative record. New York: Appleton-Century-Crofts, 1961. NOTE

1. Supplied by Modem Pet Associates, Montebello, Calif. 90640 . 